\title{
TRANSPARÊNCIA DA ÁGUA DO RESERVATÓRIO PASSO REAL E FATOR DE REFLECTÂNCIA EM IMAGENS DO SENSOR MODERATE RESOLUTION IMAGING SPECTRORADIOMETER - MODIS
}

\author{
TRANSPARENCY OF WATER PASSO REAL RESERVOIR AND FACTOR REFLECTANCE IN IMAGES SENSOR \\ MODERATE RESOLUTION IMAGING SPECTRORADIOMETER - MODIS
}

\author{
Daniela Wancura Barbieri Peixoto \\ Universidade Federal do Rio Grande do Sul (UFRGS), Rio Grande do Sul, RS, Brasil, daniwbarbieri@gmail.com \\ Waterloo Pereira Filho \\ Universidade Federal de Santa Maria (UFSM), Rio Grande do Sul, RS, Brasil, waterloopf@gmail.com \\ Felipe Correa dos Santos \\ Universidade Federal de Santa Maria (UFSM), Rio Grande do Sul, RS, Brasil, felipecorrea_rs@hotmail.com
}

\begin{abstract}
RESUMO
O trabalho teve como objetivo avaliar o uso de imagens MODIS (banda 1) para estimar a profundidade da transparência da água medida pelo disco Secchi no reservatório Passo Real. Os dados de transparência foram obtidos com a utilização do disco de Secchi em 31 estações amostrais e em duas datas (04 de julho e 28 de outubro de 2009). As imagens MODIS (MOD09) com datas mais próximas ao trabalho de campo são provenientes do Catálogo de imagens da NASA. Os valores de reflectância da banda 1, com centro de banda no comprimento de onda de $648 \mathrm{~nm}$, foram correlacionados com dados de transparência da água. A reflectância apresentou relação inversa com a transparência da água. As correlações foram significativas entre as variáveis no mês de julho, quando a transparência média foi de 1,16 metro e não foi significativa em outubro quando a transparência média foi 0,52 metro. As imagens Modis mostraram bom desempenho em condições específicas do ambiente aquático. Ao comparar dados de transparência estimada a partir da imagem de satélite com a transparência avaliada in loco, pode-se concluir que o Modis permite estimar transparências superiores a 0,5 metro.
\end{abstract}

Palavras-chave: Reservatório. Transparência da água. Reflectância. Modis. Estimativa.

\section{ABSTRACT}

This work has the goal of evaluating the use of MODIS images (band 1) to estimate the depth of the water transparence measured by the Secchi disc in the reservoir of Passo Real - RS. The transparence data were acquired by using the Secchi disc at 31 sample stations and in two different dates (July, 4th and October, 28th - both in the year of 2009). The MODIS (MOD09) images of dates close to the ones of the field works derive from the NASA image catalog. The values of band 1 reflectance - that have center of band in the wavelength of $648 \mathrm{~nm}$ - were correlated to the water transparence data. The reflectance showed an inverse relationship to the transparence of the water. The correlations were significant among the variables of the month of July, when the average transparence was 1,16 meters and it was not significant in October when the average transparence was 0,52 meter. The Modis images showed a good performance in specific conditions of the aquatic environment. When comparing satellite image estimated transparence data to the transparence evaluated in loco, one may conclude that Modis allows to estimate transparences greater to 0,5 meter.

Keywords: Reservoir. Transparence of water. Reflectance. Modis. Estimate.

Artigo recebido para publicação em fevereiro de 2015

Artigo aceito para publicação em julho de 2015

\section{INTRODUÇÃO}

O aproveitamento hidroelétrico dos rios, com a instalação de barragens, continua sendo a principal fonte de eletricidade no Brasil (REBOUÇAS, 2006). No entanto, a construção de reservatórios tem 
provocado alterações nas propriedades da água em relação ao ambiente original. O principal impacto observado é o aumento da transparência da água no sentido rio-barragem devido à deposição de material inorgânico (STEVAUX et al, 2009).

O uso de Sensoriamento Remoto permite uma abordagem sinótica do problema visto que possibilitam não apenas identificar substâncias em suspensão na água como também, a partir da análise de séries temporais, determinar seu deslocamento ao longo do tempo (JENSEN, 2009; RUDORFF, 2007). Novo et al (2007) mencionam vantagens na utilização de dados do Moderate Resolution Imaging Spectroradiometer (MODIS) em estudos aquáticos, tais como: a área de recobrimento, que possibilita estudar grandes extensões de rios e lagos; resolução radiométrica de 12 bits, que permite identificar sutis diferenças na cor da água; e, a resolução temporal diária, que possibilita estudos dos processos dinâmicos do sistema aquático com boa amostragem temporal.

Uma das maiores limitações do Sensoriamento Remoto para o estudo das propriedades ópticas dos sistemas aquáticos é a disponibilidade de imagens ópticas devido a cobertura de nuvem. A partir do lançamento dos satélites Terra e Aqua essa limitação pode ser contornada porque tornaram-se disponíveis dados do sensor MODIS, cujas imagens podem ser adquiridas com frequência diária, o que aumenta a probabilidade de obtenção de dados sem cobertura de nuvem. A grande limitação das imagens MODIS, entretanto é a resolução espacial (250 metros) nas bandas do vermelho e infravermelho, e 500 metros nas demais bandas.

O produto MOD09, oriundo do MODIS, é uma imagem da estimativa da reflectância na superfície e pode ser utilizado para estudos em sistemas aquáticos continentais, sendo seus dados computados a partir das bandas 1, 2, 3, 4, 5, 6 e 7 com centros de banda nos comprimentos de onda de 0,648, 0,858, 0,555, 1,240, 0,640, e 2,130 $\mu \mathrm{m}$, respectivamente (NOVO et al. 2007). A Tabela 1 apresenta as principais características técnicas do sensor Modis.

\begin{tabular}{|c|c|}
\hline Características & Modis - Terra \\
\hline
\end{tabular}




\begin{tabular}{|c|l|}
\hline Órbita & $\begin{array}{l}705 \mathrm{Km}, \quad \text { Sól-síncrona, quase polar } \\
\text { descendente, cruzando o equador às 10:30 } \\
\text { (horário local) }\end{array}$ \\
\hline Bandas espectrais & $\begin{array}{l}36 \text { bandas, posicionadas entre } 0,405 \text { e } \\
14,385 \mu \mathrm{m}\end{array}$ \\
\hline Resolução radiométrica & 12 bits \\
\hline Resolução espacial & $\begin{array}{l}250 \mathrm{~m} \text { bandas } 1 \text { e } 2(0,620 \text { a } 0,875 \mu \mathrm{m}) ; \\
500 \mathrm{~m} \text { nas bandas } 3 \text { a } 7(0,459 \text { a } 2,155 \\
\mu \mathrm{m}) ; \quad 1000 \mathrm{~m} \text { nas bandas } 8 \text { a } 36(0,405 \text { a } \\
8,77 \mu \mathrm{m})\end{array}$ \\
\hline Período de Revisita & \begin{tabular}{l} 
Imageamento diário, 8 e 16 dias \\
\hline
\end{tabular} \\
\hline
\end{tabular}

Tabela1. Características do sensor Modis

De acordo com suas características, as imagens MODIS são particularmente interessantes para 0 estudo de grandes reservatórios como é o caso do reservatório Passo Real. A turbidez da água pode sofrer influencia pelo escoamento superficial dos solos e pela entrada de partículas inorgânicas, tais modificações causam alterações na profundidade da zona eufótica e consequentemente na produtividade primária dos sistemas aquáticos. Uma forma de se estimar os impactos sobre a profundidade da camada eufótica é a partir da estimativa da transparência da água dos sistemas aquáticos.

O trabalho teve como objetivo avaliar o uso de imagens MODIS (banda 1), para estimar a profundidade da transparência da água medida pelo disco de Secchi no reservatório Passo Real.

\section{Metodologia}

\section{Descrição da área de estudo}

O reservatório Passo Real está localizado no alto curso do Rio Jacuí, no Rio Grande do Sul, entre as coordenadas geográficas $28^{\circ} 39^{\prime} 07$ " a $29^{\circ} 02^{\prime} 50^{\prime}$ de Latitude Sul e $53^{\circ} 03^{\prime} 14$ ” a $53^{\circ} 16^{\prime} 13^{\prime}$ de Longitude. O reservatório apresenta uma área de aproximadamente $225 \mathrm{~km}^{2}$ com uma bacia 
hidrográfica de $8.199 \mathrm{~km}^{2}$, o tempo de residência médio das águas é de 187 dias (WACHHOLZ, 2011). O reservatório foi construído com intuito de produção de energia elétrica e regulação da quantidade da água nos reservatórios à sua jusante. A presença de Latossolo Vermelho (Streck et al, 2008) e relevo colinoso na bacia hidrográfica, constante ao reservatório Passo Real, permitiu a ocupação agrícola em mais $64 \%$ da área, sendo os principais cultivos a soja no verão e o trigo no inverno (WACHHOLZ, 2011). As principais áreas da bacia hidrográfica são as dos Rios Jacuí, Rio Jacuí-Mirim e Ingaí (Figura 1). 


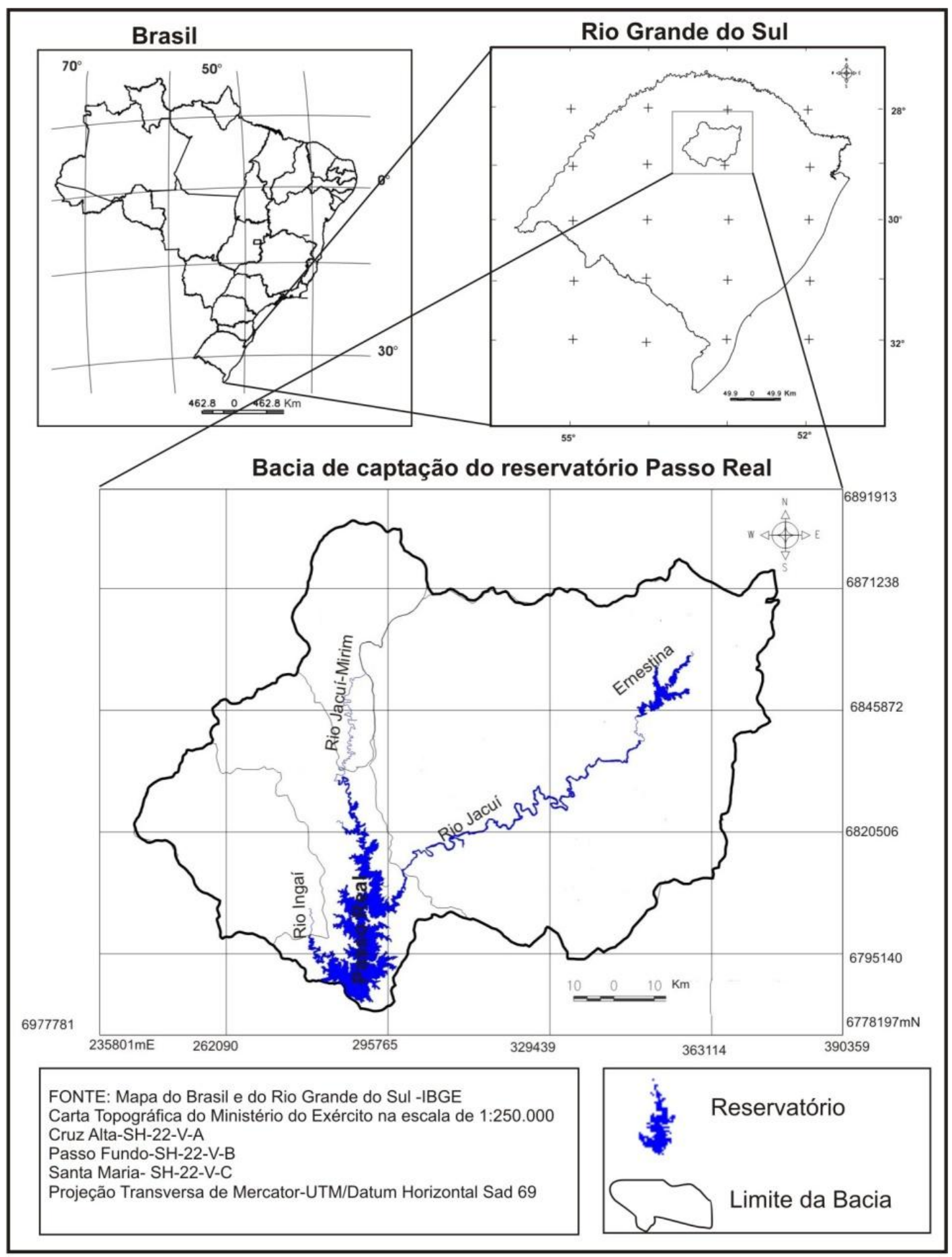

Figura 1.Localização da bacia de captação do Passo Real no Rio Grande do Sul - Brasil

\section{Medidas de Transparência da água}

Para determinar a transparência da água utilizou-se o disco de Secchi que se constitui em um disco branco de $25 \mathrm{~cm}$ de diâmetro acoplado a uma corda graduada. Para a obtenção desta medida foram 
observados fatores como: fazer as medições somente com céu claro, do lado sombreado do barco e observação sempre pela visão do mesmo pesquisador para que o resultado não fosse alterado. A medida da transparência corresponde ao desaparecimento do disco quando mergulhado na água (BARTRAN, 1996; WETZEL, 2000). Os dados foram coletados em 31 estações amostrais no reservatório Passo Real em diferentes compartimentos aquáticos distribuídos aleatoriamente a fim de abranger toda a extensão do reservatório, inclusive na entrada dos principais tributários (Figura 2). Os trabalhos de campo foram realizados nos meses de julho (dia 13) e outubro (dia 21) de 2009.

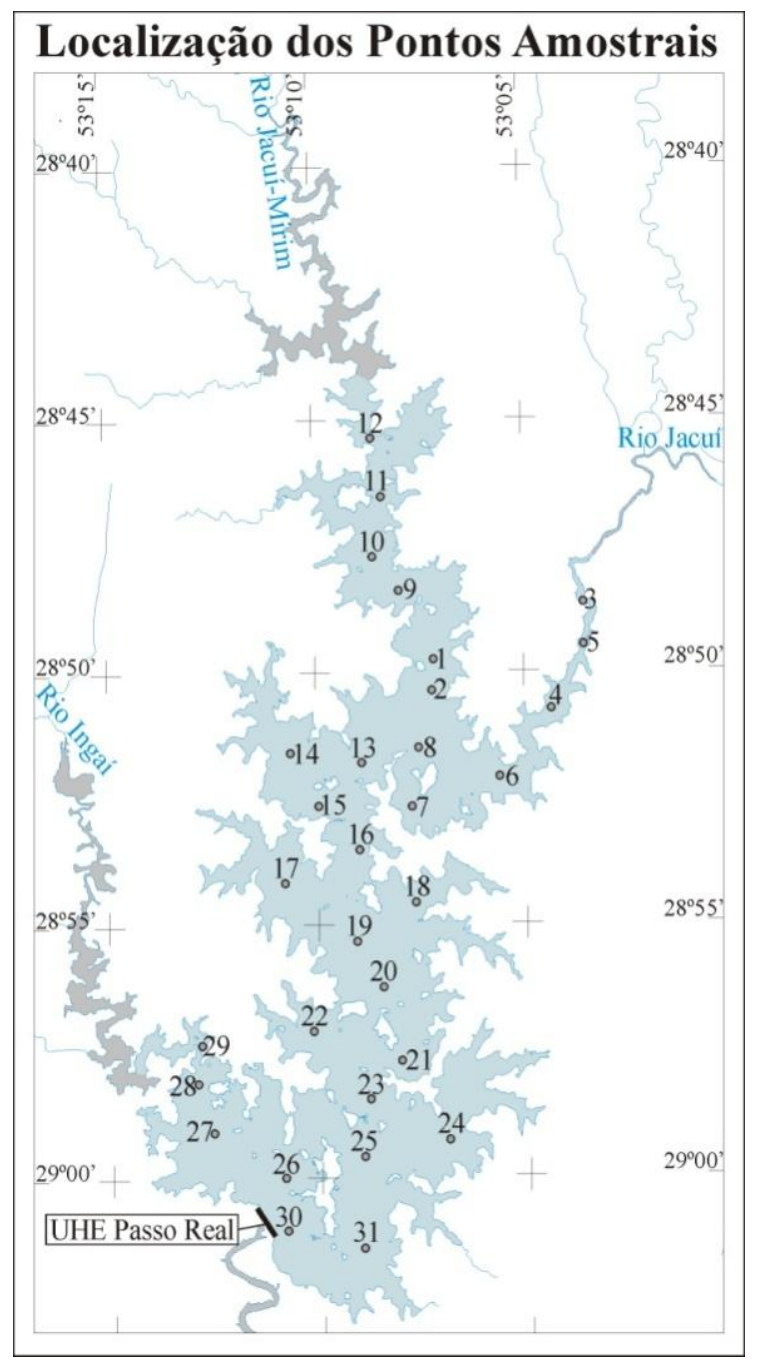

Figura 2. Localização das estações amostrais no reservatório Passo Real

\section{Reflectância da água}


As imagens de satélite do sensor MODIS foram utilizadas para obtenção dos valores de reflectância nos pontos amostrais. As estações amostrais foram identificadas nas imagens de satélite georreferenciadas a partir de suas coordenadas geográficas obtidas com o uso de GPS. O processamento das imagens foi realizado utilizando-se o programa Environment for Visualizing Imagens - ENVI versão 4.5.

O produto Modis utilizado foi o MOD09, constante ao subproduto MOD09GQ, disponibilizado no catálogo da NASA. O MOD09GQ apresenta a banda 1, correspondente ao vermelho, com resolução espacial de 250 metros. As imagens selecionadas correspondem às datas mais próximas as do trabalho de campo. Sempre foram utilizadas imagens sem cobertura de nuvens. A Tabela 2 apresenta as datas de aquisição de dados de campo e as respectivas imagens utilizadas.

\begin{tabular}{|l|c|c|c|}
\hline & Mês & Data do Trabalho de campo & Data da Imagem selecionada \\
\hline 1 & Julho & $13-7-2009$ & $04-07-2009$ \\
\hline 2 & Outubro & $21-10-2009$ & $28-10-2009$ \\
\hline
\end{tabular}

Tabela 2. Datas dos trabalhos de campo e das imagens

A banda do vermelho (banda 1) foi utilizada devido esta faixa espectral apresentar melhor resposta a presença de sólidos em suspensão (Kirk, 1994).

Especificamente no reservatório Passo Real foi observado tanto em trabalhos com reflectância da imagem do Thematic Mapper quanto em trabalhos com reflectância de campo (uso de espectrorradiômetro) que a faixa espectral correspondente ao vermelho é a que sofre maior influência dos sólidos em suspensão (PEREIRA FILHO e GALVÃO, 1997; PEREIRA FILHO et al. 2009).

\section{Técnicas estatísticas}


A técnica de correlação de Pearson foi utilizada a fim de estabelecer relação entre as variáveis, de modo que, as 31 leituras de reflectância no comprimento de onda em $648 \mathrm{~nm}$ foram correlacionadas com os 31 dados de transparência da água obtidos nos pontos amostrais.

Este método mede o quanto duas variáveis tendem a variarem juntas. A correlação de Pearson retorna um valor rs entre -1 e +1 . Para testar se o resultado $r$, formularam-se as hipóteses $h=0$ onde não há correlação e $\mathrm{h} \neq 0$ onde há correlação e considerou-se a distribuição t de Student, o qual retorna um valor $\mathrm{t}$ correspondente a um determinado nível de significância que reflete a tendência de correlação entre os dados, foram considerados níveis de significância maiores ou igual a 95\% e utilizando a tabela de valores críticos de r observaram-se os resultados, onde o valot tcal for menor ao ttab, que representa os valores críticos da distribuição t de Student, fornecido através de uma tabela pronto, pode-se afirmar que a correlação não é estatisticamente significativa. Se o tcal for maior que o ttab a correlação é significativa (CALLEGARI-JACQUES, 2003).

Callegari- Jaques ainda aponta que a relação entre as variáveis, uma função pode explicar a variação de $\mathrm{Y}$ em $\mathrm{X}$, dada por $\mathrm{y}=\mathrm{A}+\mathrm{Bx}$. A regressão linear simples também conhecida como coeficiente de determinação, é o quadrado do coeficiente de correlação e fornece o valor médio de variabilidade de uma variável em função da outra (CALLEGARI-JACQUES, 2003).

Para os dados com correlação significativa foram gerados diagramas de dispersão. Sobre os diagramas de dispersão foram ajustadas equações lineares, permitindo o cálculo do valor de regressão, que expressa a dependência entre os parâmetros analisados.

\section{Resultados e Discussão}

Os resultados mostram correlação inversa entre a transparência da água e a reflectância na região do vermelho estimada a partir da imagem MODIS. O uso da banda 1 apresentou correlação significativa para os dados do mês de julho $(-0,88)$ e não significativa no mês de outubro de 2009 considerando um 
nível de significância de 95\%. Portanto, a imagem mostrou bom desempenho para a estimativa da transparência da água quando a transparência foi maior (mês de julho com média de 1,16 metros).

As variações sazonais do Índice de Vegetação por Diferença Normalizada da área de captação durante o ano está associado a diferentes fases das culturas agrícolas. O mês de outubro corresponde a uma alta taxa de variação decrescente desse índice e, em trabalho de Wachholz (2011) foi identificada alta porcentagem de solo exposto devido ao preparo de solo para culturas de verão. Estas condições associadas à chuva e a vazão dos tributários ao reservatório proporcionaram naquele mês variabilidade na cor da água (como constatado em campo, variou de marrom a verde oliva) e menor transparência (0,52 m em média).

O mês de julho apresentou uma tendência de as maiores transparências localizarem-se em estações amostrais situadas próximas ao dique. Naquele mês as transparências superiores a 1,50 m, localizamse a jusante do ponto 19, ou seja, na segunda metade do reservatório. Este conjunto de amostras apresentou melhor ajuste a equação linear (RMS $=0,13)$. As amostras à montante da estação 17 apresentaram menor ajuste a reta $(\mathrm{RMS}=0,49)$. A Figura 3 mostra a dispersão entre a transparência da água e a reflectância na imagem.

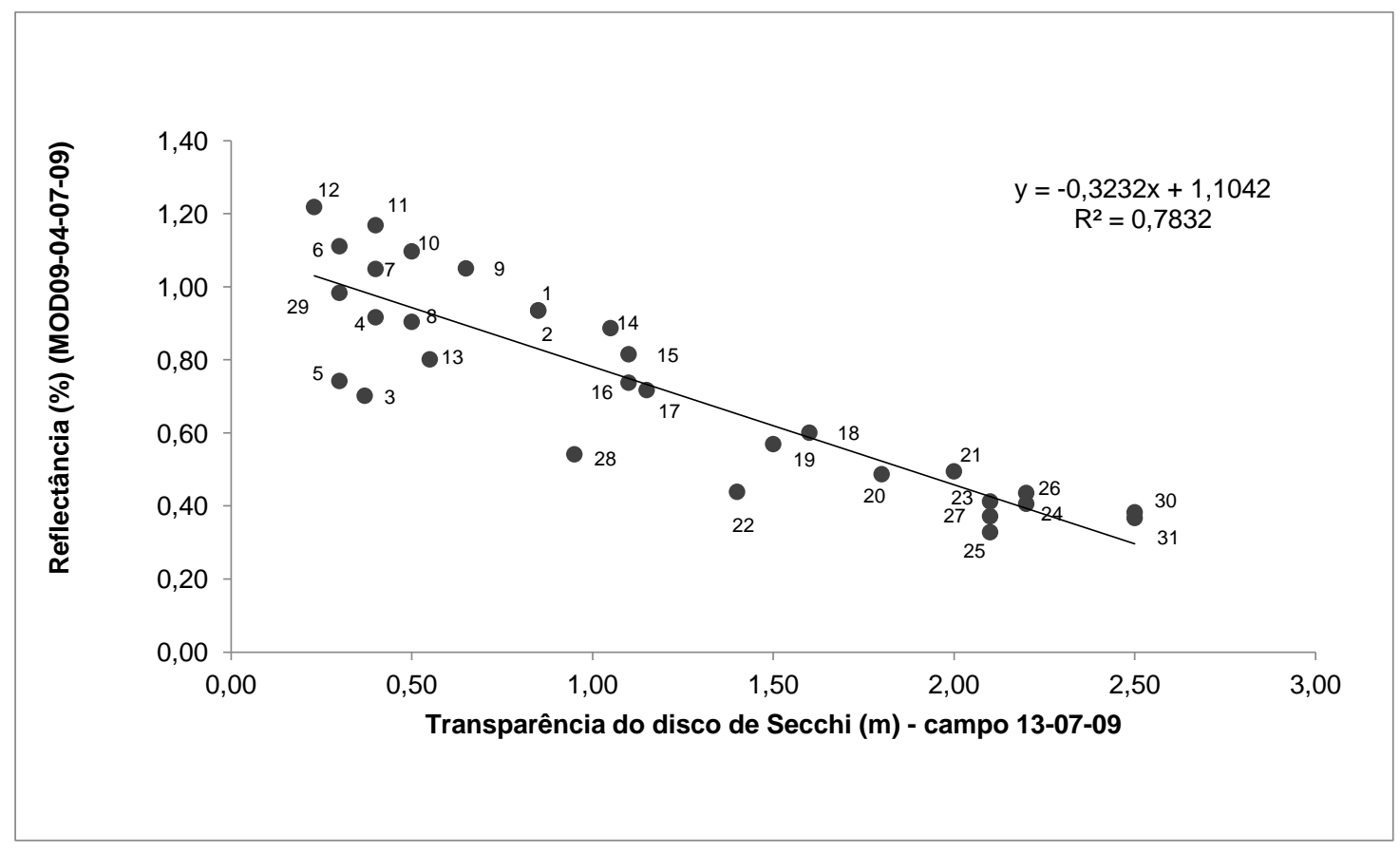


Figura 3. Relação entre dados de transparência e reflectância no vermelho no mês de julho.

A transparência estimada a partir da imagem de satélite mostrou-se coerente com a transparência avaliada in loco. A Figura 4 permite identificar os compartimentos aquáticos no reservatório com transparências semelhantes tanto nos dados de campo quanto nas estimadas pela imagem. Os setores do rio Jacuí (1), Jacuí-Mirim (2), Ingaí (3), próximo ao dique (4) e setor intermediário (5) estão enquadrados na mesma classe de transparência tanto na imagem quanto nos dados de campo. As áreas inundadas dos três tributários são identificadas com menores transparências tanto no campo quanto nas estimadas a partir da imagem. Em direção oposta é identificado o setor do dique como o de maior transparência nos dois procedimentos. O setor central do reservatório apresentou, no mês de julho, uma área de transição em relação à transparência.

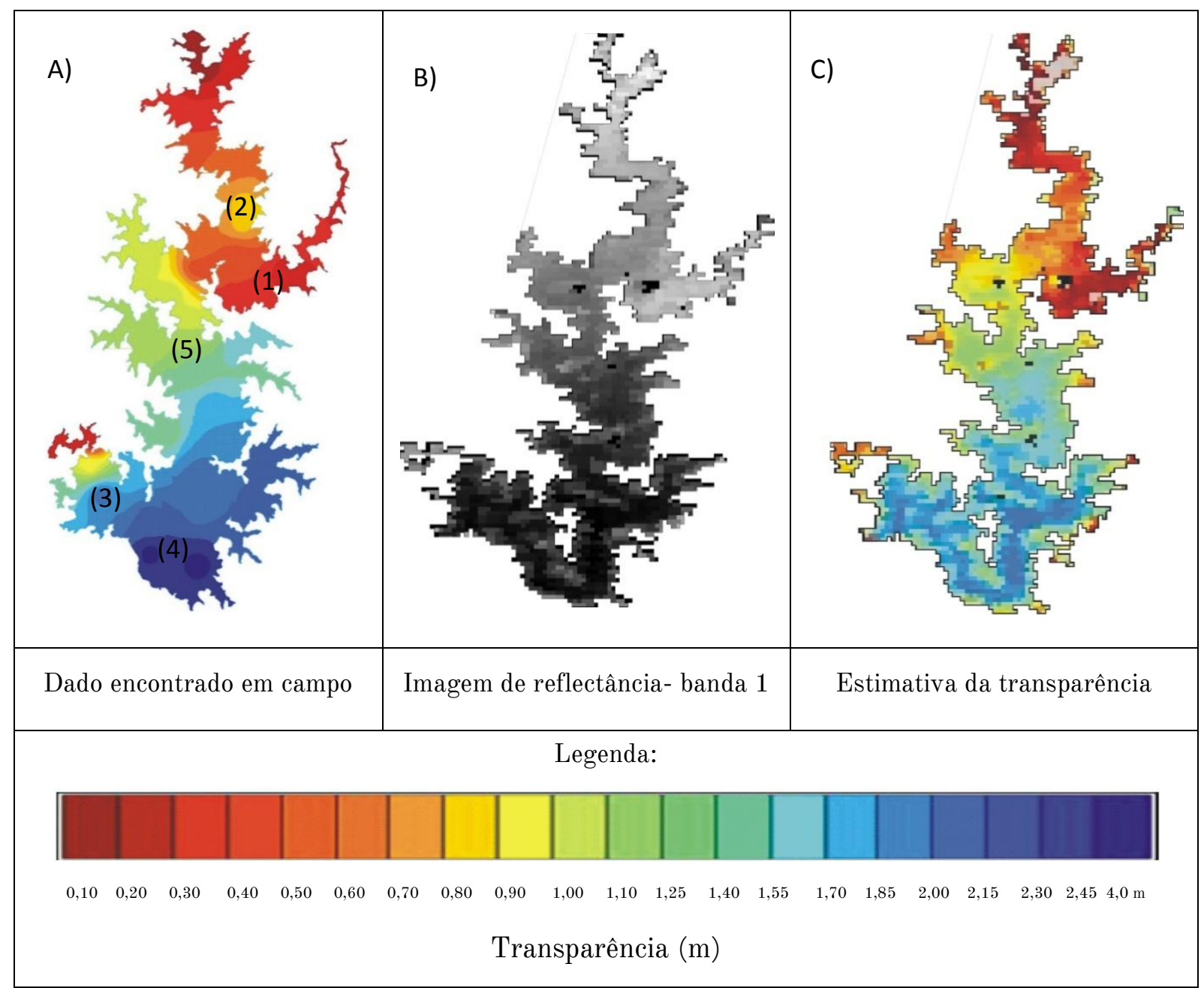

Figura 4.Transparência da água no reservatório Passo Real: A) Transparências encontradas in loco; B) Imagem de reflectância (banda 1- Modis); C) Estimativa da transparência da água a partir de dados de reflectância da imagem Modis. Isso foi para o Mês de Julho. 
Considerando somente o mês de julho observou-se, ainda, que o desempenho das imagens Modis é diferenciado em função da transparência da água. A estimativa da transparência da água não é eficiente para as transparências da água inferiores a $0,5 \mathrm{~m}$. 0 conjunto de amostras com transparências inferiores a 0,5 m não mostraram correlação significativa com a reflectância (os dados mostraram $r=-0,23)$. Por outro lado, o conjunto de amostras com transparências superiores a esta profundidade apresentou correlação significativa com $\mathrm{r}=-0,90(\mathrm{n}=24 ; \mathrm{p}=0,05)$, maior do que com o conjunto completo de dados daquele mês. Com isso observa-se que as imagens estimam melhor transparência quando estes valores são superiores a $0,5 \mathrm{~m}$ e reflectância na banda 1 inferior a $1 \%$.

O conjunto completo de dados de julho mostrou correlação significativa, porém menor $(\mathrm{r}=-0,88)$. Este resultado, embora significativo, mostra que houve uma diminuição da correlação devido à presença dos valores de transparências inferiores a $0,5 \mathrm{~m}$ (pontos localizados próximo aos principais tributários). Neste sentido, pode-se inferir que dados da banda 1 inferiores a $1 \%$ de reflectância apontam para transparências superiores a $0,5 \mathrm{~m}$ e que as mesmas podem ser estimadas a partir de informação oriunda do Modis. As reflectâncias superiores a 1\% identificam locais com transparências inferiores a $0,5 \mathrm{~m}$, mas, sob estas condições, não permitem estimar o valor de transparência com precisão.

A limitação promovida pela baixa transparência (menor que $0,5 \mathrm{~m}$ ) foi observada nas condições encontradas no mês de outubro. Naquele mês, a maioria das amostras apresentou baixa transparência (menores do que $0,5 \mathrm{~m}$ ) o que resultou em uma correlação não significativa com a reflectância. A Figura 5 mostra a dispersão dos dados entre a transparência da água e a reflectância na imagem Modis para o mês de outubro. Nesta figura observa-se que não há nenhuma estação amostral com reflectância inferior a 1\% (abaixo da linha reta), o que aponta para uma condição da água com baixas transparências, (inferiores a 0,5 m). 


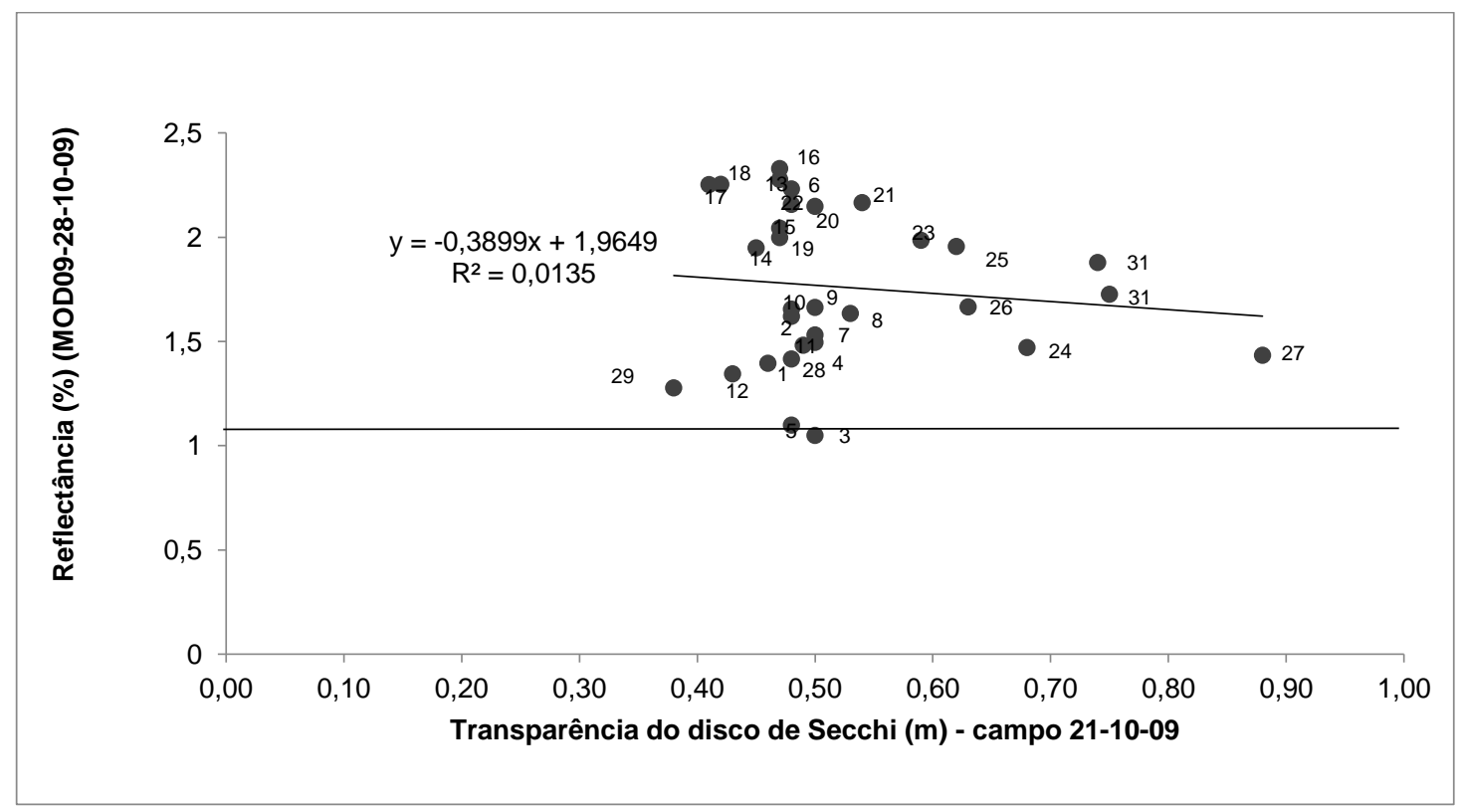

Figura 5: Relação entre dados de transparência e reflectância do Modis no mês de outubro de 2009.

\section{CONCLUSÃO}

Embora a resolução espacial de $250 \mathrm{~m}$ do sensor MODIS resulte imagens abrangendo uma grande área e, portanto, ele se mostrou compatível em estudos do reservatório Passo Real devido a sua extensa área inundada.

Com o propósito de estimar a transparência da água com uso de imagens MODIS verificou-se que a estimativa não é eficiente para aquelas transparências da água inferiores a $0,5 \mathrm{~m}$. O conjunto de amostras com transparências inferiores a $0,5 \mathrm{~m}$ não mostraram correlação significativa com a reflectância $(\mathrm{r}=-0,23)$. Por outro lado, o conjunto de amostras com transparências superiores a esta profundidade apresentou correlação significativa com $r=-0,90$. Neste sentido, pode-se inferir que dados da banda 1 inferiores a $1 \%$ de reflectância apontam para transparências superiores a 0,5 m e que as mesmas podem ser estimadas a partir de informação oriunda do MODIS.

\section{REFERÊNCIAS}

CALLEGARI-JACQUES, S. M. Bioestatística: princípios e aplicações. Porto Alegre:Artmed, 2003. 
DEKKER, A. G. Detection of optical water quality parameters for eutrophic waters by high resolution remote sensing, $\mathrm{PhD}$ Thesis. Amsterdam, Netherlands: Vrije Universiteit, 1993. 240 p.

KIRK, J. T. O. Light \& photosynthesis in aquatic ecosystems. 2nd ed. Cambridge: Cambridge University Press, 1994.

NOVO, E. M. L. M.; BARBosA, C.; FREITAS, R. M. Aplicações no Estudo de Sistemas Aquáticos Continentais. In: ShImABUKURO, Y. E.; CEBAlloS, J. C.; RUDORFF, B. F. T. (ORG.). O Sensor Modis e suas Aplicações Ambientais no Brasil. São José dos Campos: Parêntese, 2007. p. 265-276.

NOVO, E. M. L. M., Utilização de dados de Sensoriamento Remoto em Estudos Ambientais. Geografia, v. 13, 25, n. 25, p. $43-51,1988$.

PEREIRA FILHO, W.; GALVÃO, L. S. Relações entre reflectância espectral e concentração de sedimentos em suspensão no reservatório Passo Real, região Sul do Brasil. In: Simposio Latinoamericano de Percepcion Remota, 8., 1997, Mérida, Anais..., Mérida: Selper, 1997.

PEREIRA FILHO, W. et al. Influência de reservatórios em cascata nos dados de reflectância e de limnologia - Reservatórios de Passo Real e Dona Francisca, Rio Jacuí - RS. In: Simpósio Brasileiro de Sensoriamento Remoto, 14. (SBSR), 2009, Natal. Anais... São José dos Campos: INPE, 2009. p. 4813-4819. DVD, On-line. ISBN 978-85-17-00044-7. (INPE15977-PRE/10586). Disponível em: <http://urlib.net/dpi.inpe.br/sbsr@80/2008/11.17.16.19>. Acesso em: 16 fev. 2011.

RUDORFF, Conrado M. et al. Análise derivativa de dados hiperespectrais medidos em nível de campo e orbital para caracterizar a composição de águas opticamente complexas na Amazônia. Acta Amaz., Jun 2007, vol.37, no.2, p.269-280. ISSN 0044-5967JENSEN, J. R. Sensoriamento remoto do ambiente: uma perspectiva em recursos naturais. São José dos Campos, SP: Parêntese, 2009.

STEVAUX, J. C., MARTINS, D. P.; Meuer M, Changes in a large regulated tropical river: The Parana River downstream from the Porto Primavera Dam, Brazil, Geomorphology, Volume 113, Issues 3-4, Short and Long Term Processes, Landforms and Responses in Large Rivers, 15 December 2009, Pages 230-238.

STRECK, E. V. et al. Solos do Rio Grande do Sul. 2. ed. Porto Alegre: EMATER, 2008.

REBOUÇAS, A. da C. Águas Subterrãneas. In: REBOUÇAS, A. DA C; BRAGA, B; TUNDISI, J.G. Águas doces no Brasil: Capital ecológico, uso e conservação. São Paulo:2006. p. 111-144.

WACHHOLZ, F Influência das bacias hidrográficas e caracterização espaço-temporal de variáveis limnológicas em reservatório no Rio Grande do Sul. 2011.191f. Tese (Doutorado em Geografia) - Universidade Estadual Paulista, Rio Claro, 2011. 